Kansas State University Libraries

New Prairie Press

\title{
Simulation Comparison of Methods to Estimate Confidence Intervals of the Mitigated Fraction
}

Kenneth W. Wakeland

lowa State University, wakeland@iastate.edu

Brian Fergen

Boehringer Ingelheim Vetmedica Inc.

Follow this and additional works at: https://newprairiepress.org/agstatconference

Part of the Agriculture Commons, and the Applied Statistics Commons

\section{(c) (1) $\Theta($}

This work is licensed under a Creative Commons Attribution-Noncommercial-No Derivative Works 4.0 License.

\section{Recommended Citation}

Wakeland, Kenneth W. and Fergen, Brian (2017). "Simulation Comparison of Methods to Estimate Confidence Intervals of the Mitigated Fraction," Conference on Applied Statistics in Agriculture. https://doi.org/10.4148/2475-7772.1543

This Event is brought to you for free and open access by the Conferences at New Prairie Press. It has been accepted for inclusion in Conference on Applied Statistics in Agriculture by an authorized administrator of New Prairie Press. For more information, please contact cads@k-state.edu. 


\title{
Simulation Comparison of Methods to Estimate Confidence Intervals of the Mitigated Fraction
}

\author{
Kenneth Wakeland, Iowa State University \\ Brian Fergen, Boehringer Ingelheim Animal Health
}

January 18, 2018

\begin{abstract}
In the area of veterinary medicine, efficacy studies are conducted to support licensure of vaccines. Such studies are typically designed to assess a vaccine's ability to prevent or mitigate clinical disease. For example, reduction of duration/severity of clinical signs or the severity of lung lesions are often considered as primary or secondary criteria of vaccine efficacy. Studies designed to measure efficacy typically utilize two or more treatment groups and often use blocking structures to accommodate animal housing or litter related effects. When the criteria of interest are continuous or ordinal variables, as is the case with the above measurements, the mitigated fraction (MF) is often used to quantify a vaccine effect. One common approach involves determining the confidence interval for the MF using a bootstrap procedure. For data arising from studies with a blocking structure, there are two bootstrap procedures that are often used. The first resamples the blocks with replacement (randomized cluster bootstrap). The second resamples the blocks and the subjects within blocks, both with replacement (two-stage bootstrap). In addition to the bootstrap procedures, an asymptotic estimate of the variance of the MF can be calculated and used to construct a confidence interval. With three potential methods, it is of interest to determine coverage related to the associated intervals using study designs commonly used in efficacy studies. In addition, coverage was assessed in situations with and without a treatment effect. Using parameter estimates obtained from the data in which lung lesions were measured, we conducted a simulation experiment estimating the MF and confidence interval using each method described above. The results from this simulation study suggest the bootstrap procedures perform poorly when no treatment effect is present, while the confidence interval estimated using the asymptotic variance performs well. However, none of the methods perform particularly well in the presence of a treatment effect.
\end{abstract}

\section{Introduction}

Historically, label claims for biologics and pharmaceutical products are supported by the performance of the product in clinical efficacy and safety studies. For biologic studies, one 
measure of performance commonly used for continuous or ordinal variables is the Mitigated Fraction (MF) (APHIS, 2012). In keeping with the parlance of biologics studies, the MF represents the relative increase in the probability that a disease present in a vaccinated animal will be less severe than that disease present in a control animal. The MF can also be thought of as a way of measuring the overlap between two distributions. It is this interpretation that we use to motivate the mathematical definition of the MF. Let group 1 be defined as the control group and group 2 be defined as the vaccinated group. Siev (2005) defines the MF as

$$
\begin{aligned}
M F & =P\left(Y_{1}>Y_{2}\right)-P\left(Y_{2}>Y_{1}\right), \\
& =2 P\left(Y_{1}>Y_{2}\right)-1, \\
& =2 \frac{U_{1}}{n_{1} n_{2}}-1,
\end{aligned}
$$

where $Y_{1}$ represents a data point from the control group, $Y_{2}$ represents a data point from the vaccinated group, $U_{1}$ is the Wilcoxon rank sum statistic, $n_{1}$ is the number of observations in group 1 and $n_{2}$ is the number of observations in group 2. The MF defined by (1) is bounded below by -1 and above by 1 with a value of 1 signifying all values of group 2, the vaccine group, being below the values of group 1, the control group, and a value of -1 denote the opposite case. Many veterinary biologics studies will include a blocking or stratification variable, such as litter or housing. A stratified version of the MF is given by

$$
M F=2 \frac{\sum_{i=1}^{I} U_{1, i}}{\sum_{i=1}^{I} n_{1, i} n_{2, i}}-1,
$$

where the total number of strata is $I$ (Siev, 2005) is often used in these studies.

In the next section, we will examine three methods to calculate confidence intervals for the MF. These methods can be used for either MF definitions (1) or (2). In the third section we use simulation studies to examine the coverage and power of each of the methods. In the final section, we discuss the implications of the simulation studies as well as some areas of further study.

\section{Confidence Interval Calculations for MF}

The expressions (1) and (2) provide a point estimate of the MF, however, a measure of the uncertainty around that estimate is required for determination of efficacy. The confidence interval is the preferred method for representing the uncertainty of the estimate of the MF. In (Siev, 2005), a bootstrap procedure was performed to construct the confidence interval, and in the MF package available on CRAN for R (Siev, 2014), the "MFClusBoot" function performs the bootstrap procedure for stratified data, where the "boot.unit" option specifies whether or not the individuals within a strata should be resampled. Specifying "boot.unit=FALSE" results in the randomized cluster bootstrap, while "boot.unit=TRUE" results in the two stage bootstrap. The specifics of these two bootstrap procedures are described in the next 
section. This function produces two confidence intervals, a highest probability interval and a percentile based interval, we will focus our attention on the percentile based interval. In addition to the bootstrap methods, we will examine a method for constructing the confidence interval for the MF based on the asymptotic normality of the Wilcoxon statistic (Wilcoxon, 1945) (Mann and Whitney, 1947).

\subsection{Bootstrap Confidence Intervals}

Bootstrap distributions can be an attractive tool for constructing confidence intervals. However, (McCullagh, 2000) and (Field and Welsh, 2007) remind us to be cautious about how and when we apply bootstrap methods. We will make use of the terminology used in (Field and Welsh, 2007) to describe the bootstrap methods used in Siev (2014). That is, we refer to the bootstrap method in which the blocks are resampled with replacement as the randomized cluster bootstrap (RCB). To illustrate this, consider the data $Y_{i, j, k}$ consisting of $I, n_{i}$ vectors $\left\{Y_{i, 1,1}, \ldots, Y_{i, 1, n_{1, i}}, Y_{i, 2,1}, \ldots, Y_{i, 2, n_{2, i}}\right\}$. A RCB sample from this data is denoted $Y_{i, j, k}^{*}$ where $I, n_{i}$ vectors are sampled with replacement from the original data set with probability $1 / I$.

The second bootstrap method we refer to as the two stage bootstrap (2SB). As the name implies, this bootstrap has two stages of resampling, first resampling the blocks with replacement, in the same way discussed previously with the RCB. Then, we resample the observations within blocks with replacement. That is:

1. Select strata $i^{*}=1^{*}, 2^{*}, \ldots, I^{*}$ from $1,2, \ldots, I$ with replacement,

2. For each $i^{*}$, sample $n_{1, i^{*}}$ observations from $Y_{i^{*}, 1, k}$ and $n_{2, i^{*}}$ from $Y_{i^{*}, 2, k}$ with replacement.

As mentioned in the previous section, both of these bootstrapping methods are implemented in $\mathrm{R}$ package MF (Siev, 2014).

There are some concerns raised by (McCullagh, 2000) and (Field and Welsh, 2007) for these two bootstrap methods. In (McCullagh, 2000), they mention the 2SB over estimates the variability of the data and is only consistent when both $I \rightarrow \infty$ and $n_{1, i}, n_{2, i} \rightarrow \infty$. In (Field and Welsh, 2007), the authors find some higher order moments are not consistent even when the above condition holds. This is certainly a point of concern for us, even though we are considering different statistics than (Field and Welsh, 2007), we will need to keep this in mind as we explore the properties of these methods. Both (McCullagh, 2000) and (Field and Welsh, 2007) find the RCB is consistent as $I \rightarrow \infty$, though (Field and Welsh, 2007) notes sample size can be as issue for the RCB as the sample size is effectively $I$.

The bootstrap techniques are not the only methods that can be used to construct a confidence interval for the MF. In the next section, we consider the asymptotic confidence interval $(\mathrm{ACI})$ constructed using the asymptotic distribution of the Wilcoxon statistic.

\section{$2.2 \quad$ Asymptotic Confidence Interval}

The asymptotic distribution of the Wilcoxon/Mann-Whitney statistic (Wilcoxon, 1945) (Mann and Whitney, 1947) are well known to be normal under the null hypothesis case 
where $U_{1}=U_{2}$. The asymptotic normal distribution for this case has mean 0 and variance

$$
\sigma_{U}^{2}=\frac{n_{1} n_{2}\left(n_{1}+n_{2}+1\right)}{12} .
$$

Using expression (1), it is readily apparent the variance of the MF for the unstratified case would be

$$
\sigma_{M F}^{2}=\frac{4}{\left(n_{1} n_{2}\right)^{2}} \sigma_{U}^{2}
$$

The variance of the stratified case can be found using expressions (3) (2) and is given by

$$
\sigma_{M F}^{2}=\frac{4}{\left(\sum_{i=1}^{I} n_{1, i} n_{2, i}\right)^{2}} \sum_{i=1}^{I} \sigma_{U_{i}}^{2}
$$

where

$$
\sigma_{U_{i}}^{2}=\frac{n_{1, i} n_{2, i}\left(n_{1, i}+n_{2, i}+1\right)}{12} .
$$

This implies we can define the test statistic to be $z=\frac{M F}{\sigma_{M F}}$ with a corresponding confidence interval $M F \pm z_{\alpha / 2} * \sigma_{M F}$ which we will refer to as the asymptotic confidence interval (ACI).

With the introduction of these three methods for constructing confidence intervals, the natural question is what are the operating characteristics (coverage, power) for these three methods. To address these questions we perform a simulation study.

\section{Simulation Study}

The R package "MF" (Siev, 2014) that implements the bootstrap confidence interval methods discussed previously also has an example data ("piglung") set that consists of lung lesions in pigs. The lung lesion values are measured from $0-100 \%$. The litter information is also included with the data and serves as the block or stratification variable. For this analysis, there were 18 litters in which both treatments were present, each litter had between 1-5 pigs per treatment per block with a total number of 93 pigs. We use this information as the base of three simulation studies.

To perform a simulation study, we need to determine the distribution along with the corresponding parameters. To this end, we performed a logit transformation of the lung lesion data. A linear mixed model of the form

$$
\begin{aligned}
& X_{i, j, k}=\mu_{j}+\beta_{i}+\epsilon_{k(i j)}, i=1, \ldots, 18, \quad j=1,2, \\
& \beta_{i} \sim N\left(0, \sigma_{b}^{2}\right), \\
& \epsilon_{k(i j)} \sim N\left(0, \sigma^{2}\right),
\end{aligned}
$$

was performed on the logit transformed data in order to obtain estimates of variance components $\left(\sigma_{b}^{2}, \sigma^{2}\right)$ and group means $\left(\boldsymbol{\mu}=\left\{\mu_{1}, \mu_{2}\right\}\right)$. The residual diagnostics indicate this transformation was appropriate. The variance components were estimated to be $\hat{\sigma}_{b}^{2}=0.2775$ 
and $\hat{\sigma}^{2}=1.6782$ and the group means were estimated as $\hat{\boldsymbol{\mu}}=(-1.87,-2.98)$. There were 3 pigs that had lung lesion scores of exactly 0 and were excluded from the linear model. With the these mean and variance estimates, we determine the value of the MF to be 0.455 , and we can construct the simulation studies.

\subsection{Simulation Designs}

The first simulation study matches the unbalanced block structure of the original data. That is, there are 18 blocks that had the same number of observations per group per block as the original data. We generate data according to the model (5) and then apply the inverse logit transformation

$$
Y_{i, j, k}=\frac{1}{1+e^{-X_{i, j, k}}} \quad i=1, \ldots, 18, \quad j=1,2, \quad k=1, \ldots, n_{j, i} .
$$

The second and third simulation studies attempt to explore the behavior of the three methods for constructing confidence intervals as the number of blocks as well as the number of observations within a block increases. To this end, we use a similar design to the first simulation study, but with a balanced block design. That is, the model used for the second simulation study is

$$
\begin{aligned}
X_{i, j, k}=\mu_{j}+\beta_{i}+\epsilon_{i, k(j)}, & i=1, \ldots, 30, \quad j=1,2, \quad k=1, \ldots, 5, \\
\beta_{i} & \sim N\left(0, \sigma_{b}^{2}\right), \\
\epsilon_{i, k(j)} & \sim N\left(0, \sigma^{2}\right) \\
Y_{i, j, k} & =\frac{1}{1+e^{-X_{i, j, k}}}
\end{aligned}
$$

where $\sigma_{b}^{2}, \sigma^{2}$, and $\left(\mu_{1}, \mu_{2}\right)$ are the values that were estimated previously using the "piglung" data set. The only difference between the third simulation study and the second is the number of blocks changes from 30 to 100, and the number of observations per block per treatment goes from 5 to 20 .

In each of the three study designs, we use two values for $\boldsymbol{\mu}, \boldsymbol{\mu}=(0,0)(M F=0)$ and $\boldsymbol{\mu}=\left\{\mu_{1}, \mu_{2}\right\}(M F=0.455)$. We consider $\boldsymbol{\mu}=(0,0)$ to examine the performance of each of the three confidence interval construction methods under the null scenario. We now move to the results of simulations.

\subsection{Simulation Study Results}

For each design described above, 1000 data sets were generated. For each simulated data set, the three methods were each used to find the confidence interval for that data. The coverage and power were then calculated based on the theoretical values of the MF, though power was only calculated when there was a treatment difference. The type 1 error is held fixed at 0.05 for each two-sided confidence interval $(\alpha=0.05)$. 
The results for the first simulation study described above are presented in Table 1 . In the case in which there is no treatment difference $(\boldsymbol{\mu}=(0,0))$, only the asymptotic confidence interval method achieves the correct coverage. The coverage for the randomized cluster bootstrap is too low at $90.6 \%$ while the two stage bootstrap is too conservative with a coverage of $98.7 \%$. When we switch focus to the case when there is a treatment effect $(\boldsymbol{\mu}=(-2.98,-1.87))$, notice the coverages for the randomized cluster bootstrap and the two stage bootstrap remain the same, however the coverage for the asymptotic bootstrap increases to $97.7 \%$. The power for the ACI and RCB methods are approximately the same, while the power for the $2 \mathrm{SB}$ method is $14 \%$ lower than the other two methods. These results from the second simulation design, presented in Table 2, follow a similar trend. That is, the coverage for the ACI is correct in the case with no treatment effect, but is too conservative when there is no treatment effect. The coverage for the RCB is too low in both cases, and the $2 \mathrm{SB}$ is too high for both cases. The results for the final simulation design are presented in Table 3. In this case, the coverage for the RCB is close to $95 \%$ for both the treatment effect and no treatment effect cases. The ACI follows the same trend as the first two simulation designs in that the coverage is close to $95 \%$ for the case with no treatment effect and too high for the case with a treatment effect. The coverage for the 2SB increases in both the treatment effect and no treatment effect cases compared to the first two simulation experiments. This is a concerning result as we are increasing the number of blocks and number of subjects within a block and the coverage is getting more conservative, rather than decreasing to the correct value.

Table 1: Results of the simulation study for the first study design.

\begin{tabular}{cccc}
$\boldsymbol{\mu}$ & Method & Coverage & Power \\
\hline \multirow{3}{*}{$(0,0)$} & RCB & 0.906 & NA \\
& 2SB & 0.987 & NA \\
& ACI & 0.949 & NA \\
\hline \multirow{3}{*}{$(-1.87,-2.98)$} & RCB & 0.906 & 0.937 \\
& 2SB & 0.987 & 0.796 \\
& ACI & 0.977 & 0.942 \\
\hline
\end{tabular}

Table 2: Results of the simulation study for the second study design.

\begin{tabular}{cccc}
$\boldsymbol{\mu}$ & Method & Coverage & Power \\
\hline \multirow{3}{*}{$(0,0)$} & RCB & 0.926 & NA \\
& 2SB & 0.991 & NA \\
& ACI & 0.949 & NA \\
\hline \multirow{3}{*}{$(-1.87,-2.98)$} & RCB & 0.938 & 1.000 \\
& 2SB & 0.984 & 1.000 \\
& ACI & 0.974 & 1.000 \\
\hline
\end{tabular}


Table 3: Results of the simulation study for the third study design.

\begin{tabular}{cccc}
$\boldsymbol{\mu}$ & Method & Coverage & Power \\
\hline \multirow{3}{*}{$(0,0)$} & RCB & 0.935 & NA \\
& 2SB & 0.995 & NA \\
& ACI & 0.940 & NA \\
\hline \multirow{3}{*}{$(-1.87,-2.98)$} & RCB & 0.944 & 1.000 \\
& 2SB & 0.993 & 1.000 \\
& ACI & 0.973 & 1.000 \\
\hline
\end{tabular}

\section{Discussion}

The results presented in the previous section give us insight in the operational characteristics for each of the three confidence interval construction methods. However, there are some concerns with the bootstrap methods specifically. The randomized cluster bootstrap suffers from a lack of sample size. The sample size for this method is, effectively, the number of blocks present in the data. In the first simulation design, the number of blocks is 18 , the second simulation design has 30 blocks and the last has 100 blocks. We can see the coverage improve with the increase in the number blocks, however there is an impracticality to having 100 blocks. This would mean we would have to design with at least 100 litters of animals, which is not feasible for most veterinary studies.

The two stage bootstrap presents a different concern. In (Field and Welsh, 2007), they proved for the sample total the two stage bootstrap is consistent when the number of blocks $I \rightarrow \infty$ and the number of observations in a block $n \rightarrow \infty$. However, that result does not appear to hold for the MF. The results in the previous section actually appear to indicate the 2SB overestimates the variability, which we would expect given the results from (Field and Welsh, 2007) and (McCullagh, 2000), however, the width of the confidence interval increasing with the sample and block size indicates the estimate of the variability is not asymptotically consistent for the MF when using the 2SB.

The results from the asymptotic confidence interval method are mixed. The method performs well when there are no treatment effects present, which is due to the asymptotic normality of the Wilcoxon statistic under the null. However, when there is a treatment effect, the coverage of the ACI method is too high, which can be attributed to the fact that the $U_{1}$ statistic is skewed when there is a treatment effect.

If p-value is an acceptable alternative to a confidence interval, the PROC FREQ procedure in the SAS software package can perform a stratified Kruskal-Walis/van Elteren test (Van Elteren, 1960). The results of this procedure matched exactly with the ACI method, that is, when 0 was not in the confidence interval produced using the ACI, the van Elteren test rejected the null. When 0 was in the confidence interval, the van Elteren test failed to reject the null. Thus, the van Elteren test conducted in PROC FREQ can produce an equivalent analysis to the ACI method.

There is an additional option for performing the van Elteren test. The PROC NPAR1WAY 
procedure in the SAS software utilizing STAT/IML version 14.1 or later can also perform the van Elteren test. This procedure also allows for a weighted analysis in which the stratum are weighted by the number of observations in the stratum. This procedure inspires further work into each of the above methods, specifically how incorporating stratum weighting into the methods effects the results.

\section{References}

APHIS, USDA (2012). Veterinary services memorandum no. 800.202.

Field, Christopher A and Alan H Welsh (2007). "Bootstrapping clustered data". In: Journal of the Royal Statistical Society: Series B (Statistical Methodology) 69.3, pp. 369-390.

Mann, Henry B and Donald R Whitney (1947). "On a test of whether one of two random variables is stochastically larger than the other". In: The annals of mathematical statistics, pp. 50-60.

McCullagh, Peter (2000). "Resampling and exchangeable arrays". In: Bernoulli, pp. 285-301.

Siev, David (2005). "An estimator of intervention effect on disease severity". In: Journal of Modern Applied Statistical Methods 4.2, p. 14.

- (2014). MF: Mitigated Fraction. R package version 4.3.2. URL: http: //CRAN.R-project. org/package $=$ MF.

Van Elteren, PH (1960). "On the combination of independent two-sample tests of Wilcoxon". In: Bull Int Stat Inst 37.3, pp. 351-361.

Wilcoxon, Frank (1945). "Individual comparisons by ranking methods". In: Biometrics bulletin 1.6, pp. 80-83. 